$\begin{array}{cl}\begin{array}{c}\text { Revue } \\ \text { de } / \text { histoire } \\ \text { des religions }\end{array} & \text { Revue de l'histoire des religions } \\ & \begin{array}{l}\mathbf{4} 2006 \\ \text { Varia }\end{array}\end{array}$

\title{
Un syncrétisme pagano-chrétien : la glose du Pectoral d'Aaron dans le Lapidaire chrétien
}

The gloss about the gemstones of Aaron's Pectoral, from the Christian Lapidary

\section{Valérie Gontero}

\section{(2) OpenEdition}

\section{Journals}

Édition électronique

URL : http://journals.openedition.org/rhr/5212

DOI : 10.4000/rhr.5212

ISSN : 2105-2573

Éditeur

Armand Colin

\section{Édition imprimée}

Date de publication : 1 décembre 2006

Pagination : 417-437

ISBN : 978-2-2009-2106-4

ISSN : 0035-1423

\section{Référence électronique}

Valérie Gontero, «Un syncrétisme pagano-chrétien : la glose du Pectoral d'Aaron dans le Lapidaire chrétien », Revue de l'histoire des religions [En ligne], 4 | 2006, mis en ligne le 29 janvier 2010, consulté le 19 avril 2019. URL : http://journals.openedition.org/rhr/5212 ; DOI : 10.4000/rhr.5212 


\section{Un syncrétisme pagano-chrétien : la glose du Pectoral d'Aaron dans le Lapidaire chrétien}

Le Lapidaire chrétien, qui décrit et commente les douze gemmes du Pectoral d'Aaron et de la Jérusalem Céleste, est un texte bipartite d'environ mille trois cents vers (version du ms. M). La première partie, païenne, décrit les douze gemmes et leurs vertus, dans la lignée du De Lapidibus de Marbode de Rennes. La seconde partie, chrétienne, reprend chaque pierre pour en donner la signification symbolique et religieuse, une glose qui procède par analogie en se fondant sur les couleurs, les nombres, etc. Notre étude vise à mettre au jour cette glose complexe et syncrétique, qui met en cuvre des connaissances théologiques précises tout en réinterprétant les croyances païennes.

\section{The gloss about the gemstones of Aaron's Pectoral, from the Christian Lapidary}

The Christian Lapidary, in which are described the twelve gemstones of Aaron's Pectoral and of the Celestial Jerusalem, is a bipartite text of about one thousand and three hundred verses (version of manuscript $M$ ). In the first part, which is pagan, are described the twelve gemstones and their properties, following the tradition of Marbode's De lapidibus. In the second part, which is Christian, for each gemstone, are given the symbolic and religious meaning, that is to say a gloss which uses analogies based on colours, numbers and so on. The aim of my study is to show this complex and syncretistic gloss which uses precise theological knowledge and gives a new interpretation of pagan believes. 
Structure la plus simple de l'exégèse, la glose désigne à l'origine le synonyme donné pour un mot du texte biblique, afin d'en éclairer le sens : il s'agit d'une explication lexicale ${ }^{1}$. Guillaume de Conches la distingue ainsi du commentaire : «Le commentaire /.../ s'en tient à la sententia, sans se préoccuper du fil de la lettre ou de l'explication de cette lettre. La glose, elle, s'attache à tout celà ; d'où le nom de « glose », c'est-à-dire « langue » : elle doit en effet expliquer en clair, comme si la langue elle-même du docteur s'exprimait de vive voix $»^{2}$.

Brève à ses débuts, la glose connaît une amplificatio aboutissant à la "glose ordinaire », matérialisée par la mise en page du texte biblique, réduit souvent à une colonne et enrichi de gloses marginales et interlinéaires. Au XII siècle, cette glose devient la méthode de prédilection de l'exégèse des écoles, située historiquement entre les exégèses monastique et universitaire. Réfutant les quatre sens définis par Henri de Lubac ${ }^{3}$, Gilbert Dahan distingue deux niveaux dans l'exégèse biblique : la lettre et l'esprit ; la progression de l'une à l'autre passe par la translatio ${ }^{4}$. À chaque niveau correspondent

1. Pour une approche moderne de la glose, consulter le recueil d'articles Le mot et sa glose, sous la direction d'Agnès Steuckart et Aino Niklas-Salminen, Aix-en-Provence, Publications de l'Université de Provence, 2003 (langues et langage, $\left.\mathrm{n}^{\circ} 9\right)$.

2. Guillaume de Conches, Glosae super platonem, éd. E. Jeauneau, Paris, 1965, p. 67, texte traduit et cité par Guy Lobrichon, «Une nouveauté : les gloses de la Bible », dans Le Moyen Age et la Bible, dir. Pierre Riché et Guy Lobrichon, Beauchesne, Paris, 1984 (Bible de tous les temps 4), p. 95-114, en particulier p. 96.

3. À savoir les sens littéral, allégorique, tropologique (moral) et anagogique (mystique), selon l'ouvrage Exégèse médiévale. Les quatre sens de l'Écriture, Paris, 1959-1964, 4 volumes.

4. Les sens historique et rhétorique de la translatio s'enrichissent d'une acception propre à l'exégèse : " D'une part, le message divin (transcendant) est traduit, transposé, transféré dans un texte humain : par exemple, pour énoncer une qualité éminente de la divinité, sera utilisée l'expression "Dieu est juste", avec donc le terme "juste", dont nous, hommes, comprenons le sens à notre niveau (je pense à un juge, à Salomon, à un homme de bien) ; mouvement descendant ici, qui va vers l'homme. Mais celui-ci ne devra pas se contenter de cette signification humaine, immédiate ; il y aura donc, d'autre part, un mouvement ascendant, par lequel l'homme tentera de trouver, dans le mot humain "juste", une réalité suréminente, présente absolument en Dieu, en qui 
différentes catégories de gloses $\left.{ }^{5}: 1\right)$ pour l'exégèse littérale : les gloses « littéraires » ou figures de rhétorique ; les gloses historiques et archéologiques ; plus rarement, les gloses théologiques ou philosophiques ; 2) pour l'exégèse spirituelle : l'allégorie ; la tropologie ; plus rarement l'anagogie.

Les strates de signification du texte biblique, du sens littéral au sens spirituel, sont progressivement mises au jour ; cette méthode s'applique corollairement aux réalités de la Création, considérées comme des signes disposés par Dieu. Comme le souligne George Duby : «Les mots, la nature, tels sont bien en effet les deux champs, accessibles à l'esprit humain, où Dieu consent à se manifester. Par conséquent le moine scrute l'Ecriture, et tout l'enseignement de la grammaire le met en mesure de pénétrer par degrés, en progressant du textuel au spirituel, le sens de chacun de ces vocables. Il scrute aussi le monde créé, en quête de ces analogies dont la chaîne continue peut le conduire vers la lumière $»^{6}$.

\section{GLOSE ET LAPIDAIRE CHRÉTIEN}

À partir de ce postulat s'est développée une littérature didactique dont le but est d'expliquer et d'expliciter ce va-et-vient exégétique : les encyclopédies, à l'ambition totalisante ; les bestiaires et les lapidaires, dont l'exégèse se limite aux domaines animal et minéral.

La tradition lapidaire des $\mathrm{XII}^{\mathrm{e}}$ et $\mathrm{XIII}^{\mathrm{e}}$ siècles présente trois types de textes ${ }^{7}: 1$ ) les lapidaires magiques ou astrologiques, qui étudient

elle coexiste avec d'autres aspects infinis, sans que Son unité se trouve brisée », L'exégèse chrétienne de la Bible en Occident médiéval (XII -XIVe siècles), Paris, Éditions du Cerf, 1999 (Patrimoine Christianisme), p. 46.

5. Idem, p. 126-127. Sur la glose, voir les pages 123 à 129.

6. Le Moyen Age. Adolescence de la chrétienté occidentale. 980-1140, Genève, Skira, 1995, p. 179-180.

7. Voir la mise au point de Michel Salvat, « Du pectoral d'Aaron aux lapidaires médicaux : l'infini pouvoir des pierres », dans Nature et Encyclopédies, Actes du Colloque d'Alençon, réunis et édités par D. Hüe, 6-7 avril 1991, p. 205-218. Aux siècles suivants, la typologie des lapidaires se complexifie, voir l'introduction de l'ouvrage de Camillo Leonardi, Les pierres talismaniques 
les gemmes dans leurs rapports avec les métaux et les planètes ; 2) les lapidaires scientifiques, qui donnent leurs vertus, dans la lignée du De Lapidibus de Marbode de Rennes, qui constitua, pour le Moyen Âge, «le code anonyme de la minéralogie médicale et phylactérique $»^{8} ; 3$ ) les lapidaires chrétiens, sur lesquels va porter notre étude. L'appellation «lapidaire chrétien» est, à mon sens, inadéquate : elle camoufle en effet la composition bipartite et mixte de ce type de lapidaire. La première partie, d'inspiration païenne, énonce, pour chacune des gemmes, les vertus tirées du De Lapidibus de Marbode. Seule la seconde partie est proprement chrétienne: énumérées à nouveau, les pierres font l'objet d'une exégèse spirituelle, dans la lignée de Bède et d'Albert le Grand ${ }^{9}$.

Le lapidaire mixte, mi-chrétien, mi-païen, est facilement identifiable par son incipit : Cil qui aime pierres de pris. Léopold Pannier situe la composition de ce traité : " au second quart du XIII" siècle, dans la première partie du règne de Louis IX, époque d'où date, dans la littérature française, toute une série d'ouvrages pieux ${ }^{10}$.

Anonyme, ce texte est conservé dans sept manuscrits - les six manuscrits recensés par Léopold Pannier ${ }^{11}$ et dont il a fourni une édition composite en 1882, auxquels il faut ajouter le manuscrit de la Bibliothèque municipale Méjanes d'Aix-en-Provence, manuscrit du XIV ${ }^{\mathrm{e}}$ siècle, présenté par Charles Joret en appendice de cette édition ${ }^{12}$.

(Speculum lapidum, livre III), texte, traduction, commentaire et annotations par C. Lecouteux et A. Monfort, Paris, Presses de l'Université de Paris-Sorbonne, 2002, p. 7-22.

8. Expression empruntée à Léopold Pannier, Les lapidaires français du Moyen Age des XII ${ }^{e}$ XIII et XIV siècles, réunis, classés et publiés accompagnés de préfaces, de tables et d'un glossaire, Paris, Vieweg, 1882 (BEHE), p. 5.

9. Sur l'étude des sources, consulter l'ouvrage de Léon Baisier, The Lapidaire Chrétien. Its Composition, its Influence, its Sources. A Dissertation, Washington, The Catholic University of america, 1936.

10. Les lapidaires français du Moyen Age des XII ${ }^{e}$, XIII et XIV siècles, op. cit., p. 231.

11. BnF. fr 14964 ; BnF. fr. 24428 ; BnF. fr. 14970 ; BnF. fr 2008 ; ms. 51 de la Bibliothèques de Chartres ; ms. Sloane 413 du British Museum (idem, p. 228-237).

12. Ibidem, p. 299-302. 
Ce manuscrit, désigné par la lettre $\mathrm{M}$, constituera mon manuscrit de référence ${ }^{13}$.

Le lapidaire mixte est consacré aux douze gemmes les plus célèbres de La Bible : celles du Pectoral d'Aaron, reprises en partie par la Jérusalem Céleste. L'Exode décrit deux fois le gigantesque pendentif qui orne la poitrine du Grand Prêtre, élaboré selon les directives divines : «Ils le garnirent de quatre rangs de pierres précieuses : première rangée : une sardoine, une topaze, une émeraude ; deuxième rangée : un rubis, un saphir, un jaspe ; troisième rangée : une pierre d'ambre, une agate et une améthyste; quatrième rangée : une chrysolite, un onyx et un béryl ; elles étaient serties d'or dans leurs montures ${ }^{14}$.»

À la fin de la Bible, L'Apocalypse de saint Jean dépeint la Jérusalem céleste, dont les piliers sont taillés dans les mêmes gemmes, à quelques exceptions près : «Les soubassements du mur de la ville sont ornés de toutes sortes de pierres précieuses; le premier est de jaspe ; le deuxième de saphir ; le troisième de calcédoine ; le quatrième d'émeraude; le cinquième de sardonyx ; le sixième de sardoine; le septième de chrysolithe ; le huitième de béryl, le neuvième de topaze; le dixième de chrysoprase ; le onzième d'hyacinthe ; le douzième d'améthyste (XXI, 19-20). »

\section{Tableau ANALYTiQue de La ParTiE ChrÉtienNe}

Dans le lapidaire, l'exégèse s'effectue à plusieurs niveaux, de la partie au tout, du microcosme au macrocosme. Ainsi une senefiance est dévolue à chaque gemme, à chaque rangée (parfois même au rang de la gemme sur la rangée) et enfin à l'ensemble des douze

13. Ma transcription de ce manuscrit est consultable sur le site du CETM de l'Université de Rennes 2 - Haute Bretagne : <http://www.uhb.fr/alc/medieval/ lapidaire/Lapidairechretien.htm>.

14. Voir aussi Exode, XXXIX, 8-13. Pour la Bible et sa traduction, nous utilisons l'édition suivante : La Sainte Bible, texte latin et traduction française d'après les textes originaux avec un commentaire exégétique et théologique, commencée sous la direction de L. Pirot, continuée sous la direction d'A. Clamer, Paris, Letouzey \& Ané éditeurs, 1936-1956, 12 tomes. 
pierres du Pectoral. Le texte considère les qualités physiques et la disposition des gemmes pour établir des correspondances avec les qualités morales et les expériences spirituelles des chrétiens, comme l'illustre le tableau suivant, récapitulant les données de la seconde partie du lapidaire.

Quel est le vocabulaire utilisé pour dire la glose ? Le mot glose lui-même est utilisé à cinq reprises ${ }^{15}$. Il apparaît même dans son sens premier, celui de synonyme, d'équivalence lexicale dans l'article sur l'ambre :

Li livres nos dit et espont

la beste qui ses reins repont

por ligure qu'elle tant einme,

Moyses buof la beste cleinme,

en la glose est einsi clamée ;

(et) es lapidaires est nomée

linz ceste beste (v. 1027-1033)

Signification apparaît trois fois dans le corps du texte, présence exacerbée par l'utilisation de ce nom dans les treize rubriques de la seconde partie ${ }^{16}$. Il est concurrencé par les dix occurrences de senefiance, terme d'autant plus important qu'il clôt le lapidaire ${ }^{17}$. Les verbes senefier et ramentevoir sont également fréquents. La récurrence de ce vocabulaire est illustrée par ces vers sur le saphir :

Seint Jehan vit secondement

ceste pierre ou haut fondement

ou elle fu seconde mise ;

la seconde vertu promise

senefia : c'est esperance,

et por ceste senefiance

fu mis en la seconde tire

d'Aaron ; li safirs nos tire

et ramentoit la grant hautece

du ciel, que par notre paresce

perdons a avoir /.../ (v. 963-973)

15. Le terme glose apparaît cinq fois, aux vers 921, 1031, 1065, 1208, 1224.

16. Vers $677,1058,1266$.

17. Vers 664, 676, 704, 750, 920, 939, 968, 1241, 1281, 1300. 


\begin{tabular}{|c|c|c|c|c|}
\hline 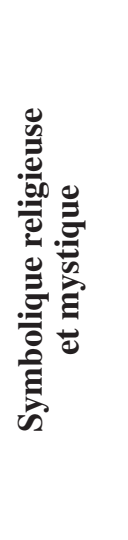 & & 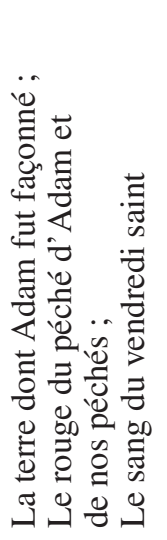 & 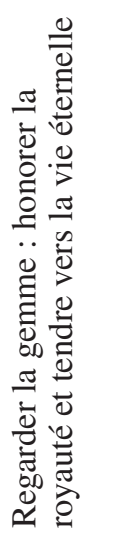 & 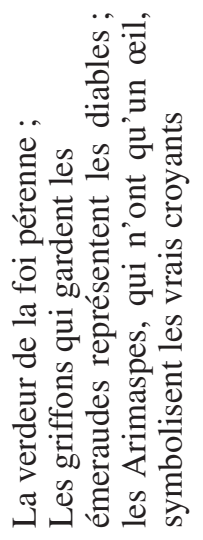 \\
\hline$\underset{\partial}{ٍ}$ & & $\begin{array}{l}\stackrel{0}{0} \\
\stackrel{0}{\Xi} \\
0\end{array}$ & 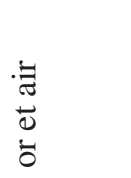 & $\stackrel{\frac{0}{0}}{>}$ \\
\hline 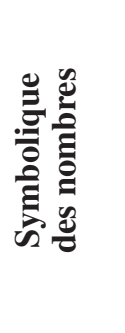 & & 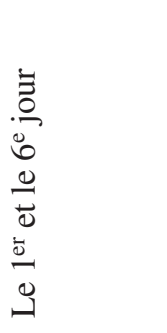 & 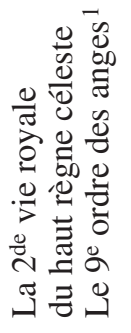 & 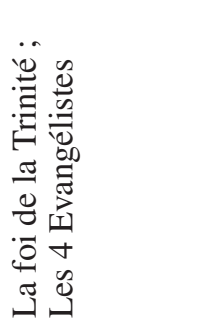 \\
\hline 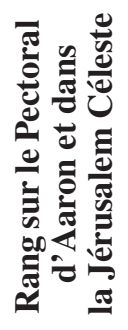 & $\begin{array}{l}0 \\
0 \\
0 \\
0 \\
\text { है } \\
0 \\
0 \\
0\end{array}$ & $\stackrel{\Xi}{e}$ & $\frac{\text { จั }}{\text { ปे }}$ & $\frac{0}{\stackrel{0}{d}}$ \\
\hline 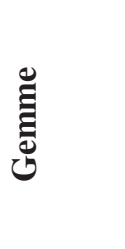 & 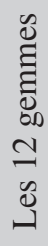 & 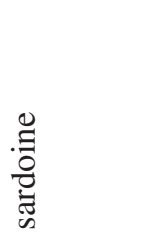 & 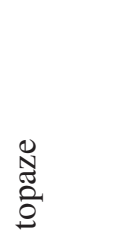 & 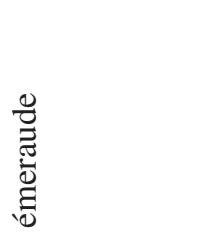 \\
\hline
\end{tabular}




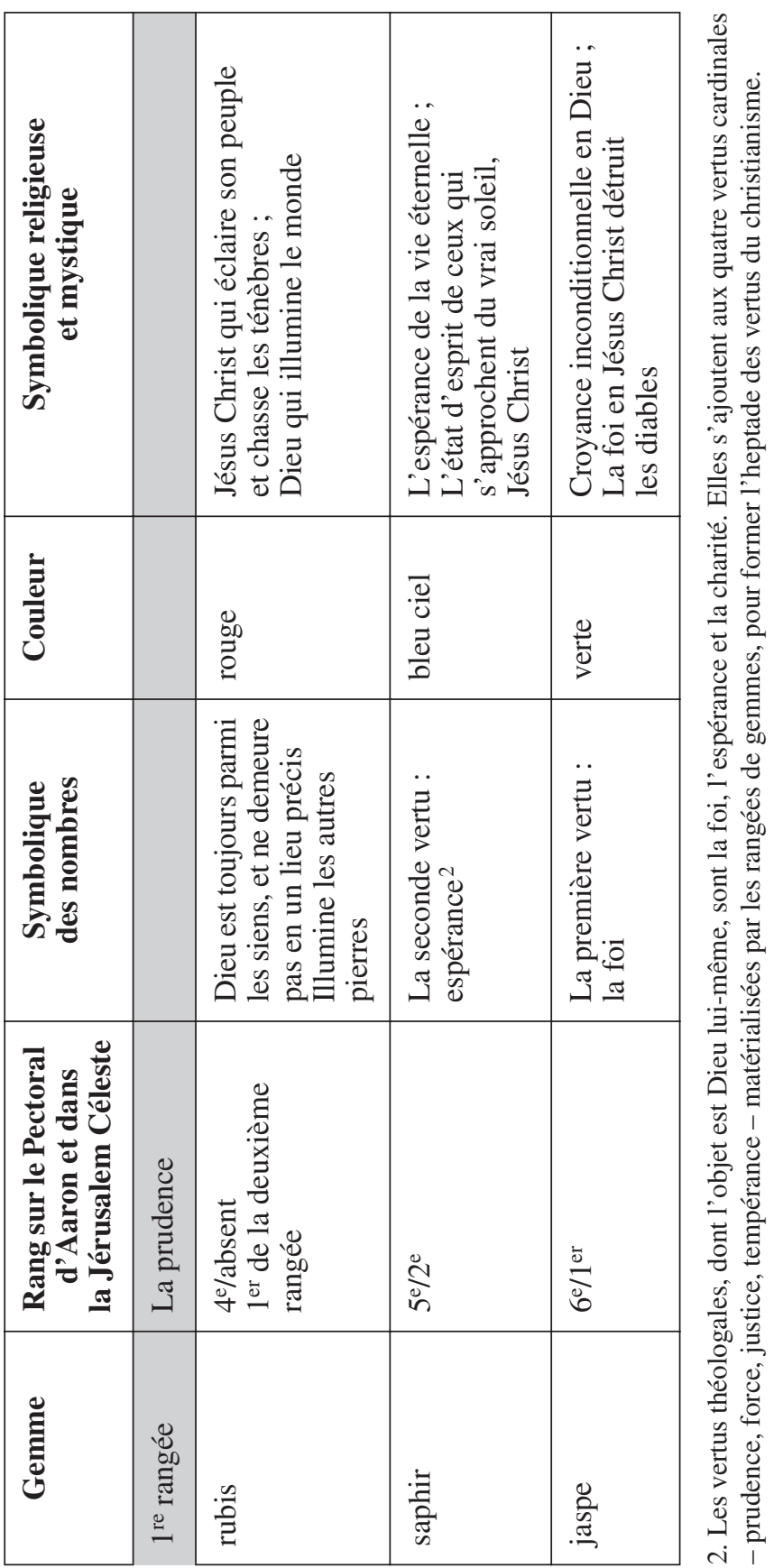




\begin{tabular}{|c|c|c|c|c|}
\hline 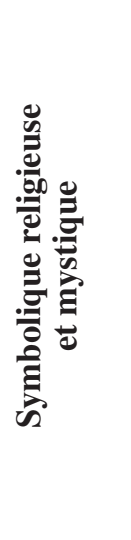 & & 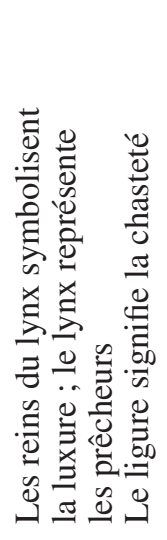 & 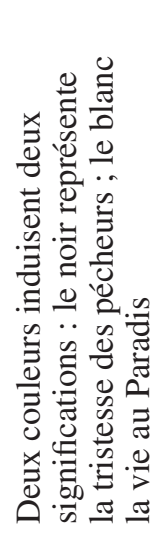 & 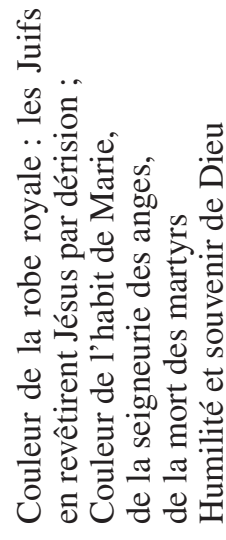 \\
\hline 光 & & 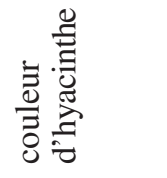 & : & $\begin{array}{l}0 \\
\stackrel{0}{\Xi} \\
0 \\
0\end{array}$ \\
\hline 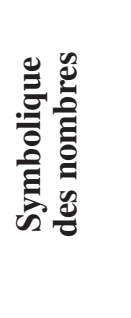 & & 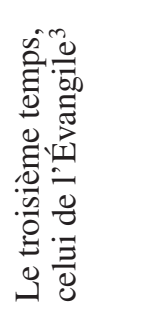 & 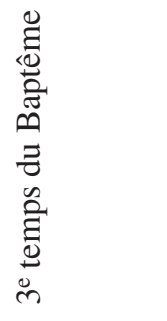 & \\
\hline 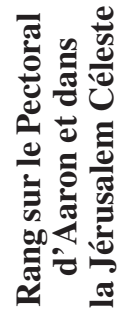 & 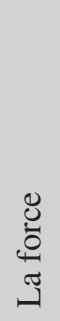 & 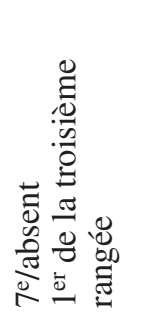 & 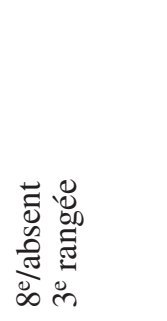 & $\begin{array}{l}\overrightarrow{0} \\
\text { है } \\
\frac{\tilde{\sigma}}{\sigma} \\
\delta\end{array}$ \\
\hline 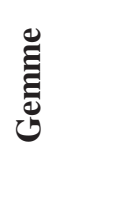 & 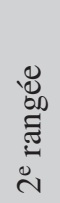 & 苛 & 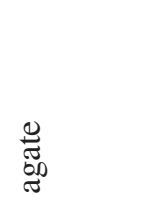 & 怘 \\
\hline
\end{tabular}

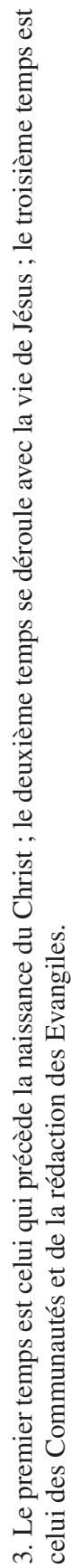




\begin{tabular}{|c|c|c|c|}
\hline 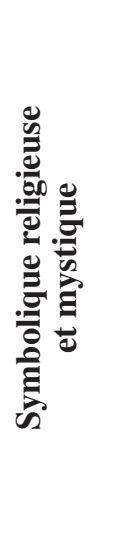 & & 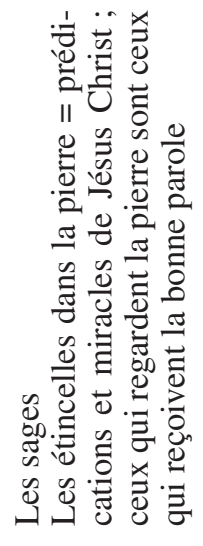 & 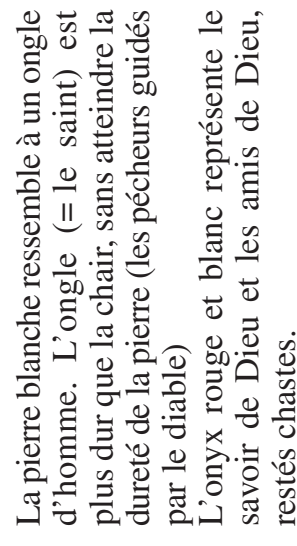 \\
\hline 党 & & 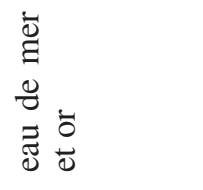 & 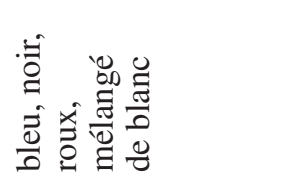 \\
\hline 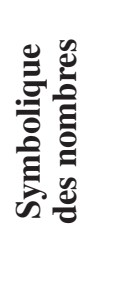 & & 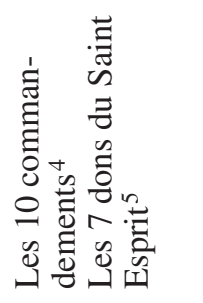 & 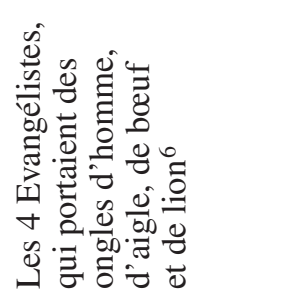 \\
\hline 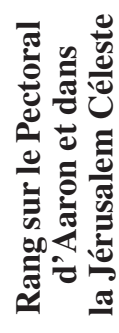 & 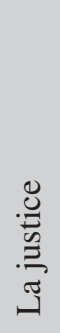 & $\frac{\stackrel{0}{5}}{0}$ & 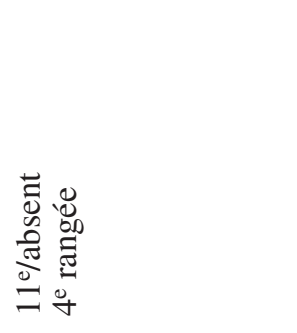 \\
\hline 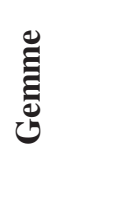 & 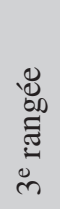 & 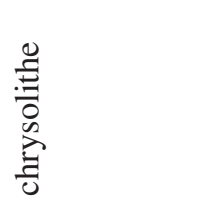 & 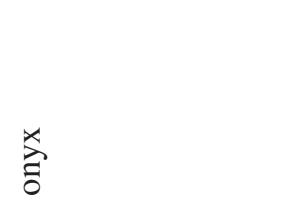 \\
\hline
\end{tabular}

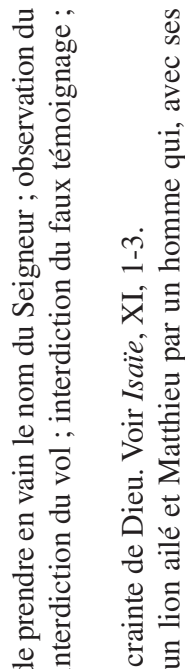

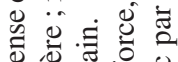

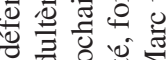

․ㅡㄴ

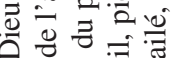

즐.

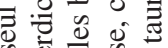

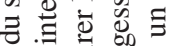

잉

흏월

.

ते

․․…

:

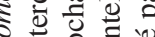

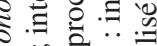

常表

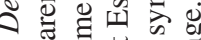

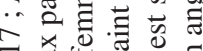

光合语

ㅋㅛㅖ

$x+y$ : 0

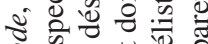

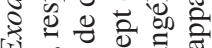

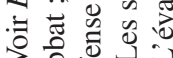

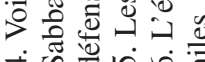




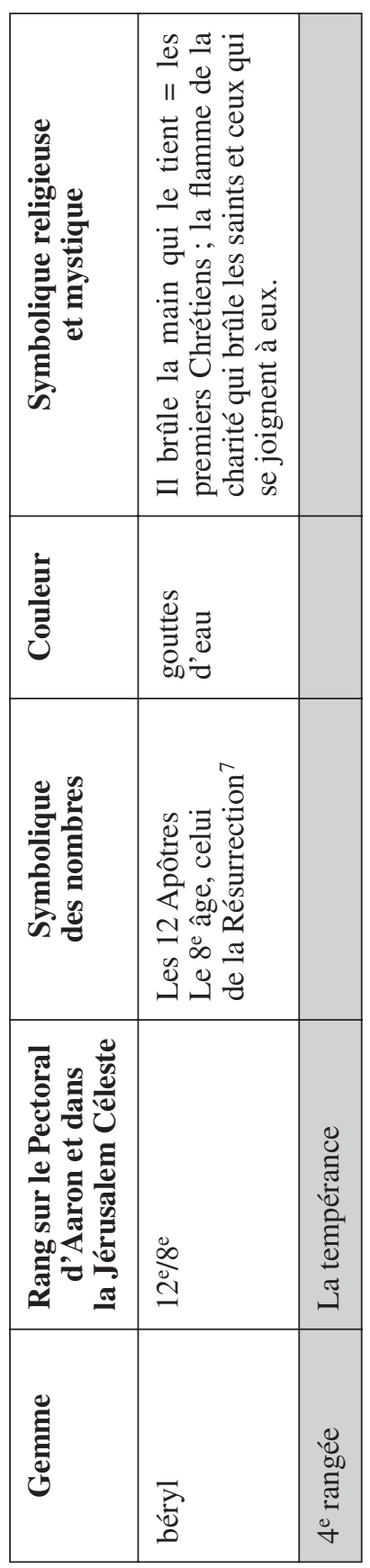

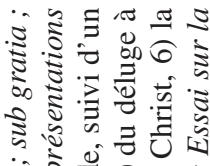

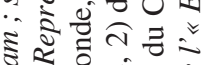

宊

के

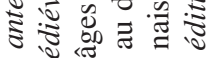

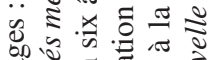

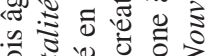

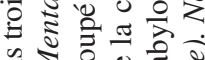

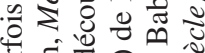

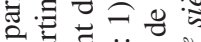

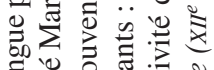

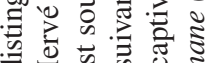

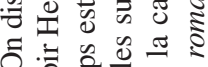

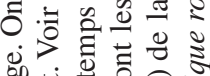

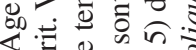

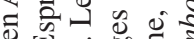

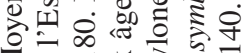

$\sum 0 \dot{0} \frac{\vec{a}}{2} \dot{0}$

ส

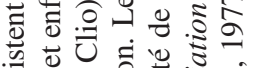

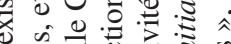

ठำ

๑ 引

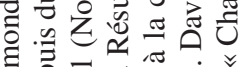

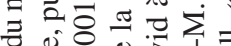

की

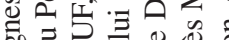

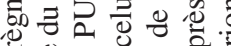
品 के ने ฮ ๑े.

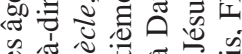

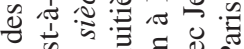

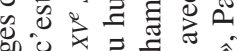

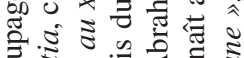

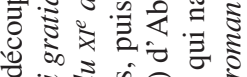

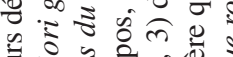

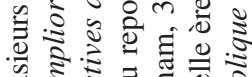
至 人 


\section{LA GLOSE PAR ANALOGIE}

Cette myriade de senefiances est engendrée par un unique principe directeur : $l^{\prime}$ analogie ${ }^{18}$. À partir d'un point commun, ténu ou plus frappant (nombre, couleur, particularité, etc.), le lapidaire construit une passerelle entre la gemme et la doctrine chrétienne, et tisse ainsi l'écheveau de la glose. L'approche analogique est résolument empirique : l'observation de la réalité minérale est le point de départ de l'interprétation, qui transcende les données premières : la semblance est le tremplin de la senefiance; ces deux noms apparaissent à la rime, à la fin de l'article sur la sardoine :

Ce fu la premiere semblance

des .XII. et la senefiance. (v. 749-750)

Le raisonnement analogique, qui sous-tend l'ensemble du texte, s'appuie systématiquement sur le nombre et sur la couleur, mais reprend aussi des propriétés décrites dans la partie païenne du lapidaire. L'analogie principale, aux ramifications variées, s'enrichit parfois d'analogies secondaires, qui étoffent et complexifient la glose.

L'analogie par le nombre, véritable mode de pensée au Moyen Âge, est la plus marquante dans le lapidaire. Les clercs accréditent l'exégèse biblique des nombres en se fondant notamment sur le verset suivant : "Mais vous réglez toutes choses avec mesure, avec nombre et avec poids » (Sagesse, XI, 21).

Dans son versant numérique, la glose s'attache au rang de la gemme, sur le pectoral d'Aaron (rang parmi les douze gemmes, rangée, place sur la rangée) et dans la Jérusalem céleste (rang parmi les piliers de gemmes). Pourquoi douze gemmes? Le douze représente le syncrétisme du nombre matériel 4 et du nombre spirituel 3, et fait écho au 7, qui incarne la perfection. Les gemmes matérialisent les douze tribus d'Israël - comme il est dit dans L'Exode et rappelé dans le lapidaire (v. 601-608) - mais aussi les douze apôtres (v. 662-667).

18. Comme le souligne Marie-Madeleine Davy, « les analogies qui relient le macrocosme au microcosme forment le fondement du symbolisme médiéval et expliquent l'importance donnée à l'univers, puisque de la nature apparaît le miroir dans lequel l'homme peut contempler l'image de Dieu », Initiation à la symbolique romane (XII siècle), op. cit., p. 159. 


\begin{tabular}{|c|c|c|c|}
\hline Rang & Pectoral d'Aaron & Apôtre & Tribu d'Israël \\
\hline 1 & sardoine & Matthieu & Judah \\
\hline 2 & topaze & Pierre & Simon \\
\hline 3 & émeraude & Barthélémy & Ephrä̈m \\
\hline 4 & rubis & André & Ruben \\
\hline 5 & saphir & Philippe & Issachar \\
\hline 6 & jaspe & Jacques le Majeur & Zabulon \\
\hline 7 & ambre & Matthias & Nephtali \\
\hline 8 & agate & Judas & Dan \\
\hline 9 & améthyste & Jean & Gad \\
\hline 10 & chrysolithe & Thaddée - Simon & Asher \\
\hline 11 & onyx & Jacques le Mineur & Benjamin \\
\hline 12 & béryl & Thomas & Manassé \\
\hline
\end{tabular}

Le poète établit également un double parallélisme entre les gemmes et les pierres, les apôtres et les saints : le premier groupe l'emporte sur le second, par la volonté de Dieu.

Mes seinte escripture nos dit, qui nos tesmoigne en cest escrit, que Dieus, qui fit la paternostre, mist pooir sur chacun apostre, et pootez leur dona meintes ; si fit il aus seinz et aus seintes, mes la seignorie enporterent li apoustre qui Dieu amerent : autresi sunt les .XII. james sur les pierres seignors et dames. (v. 689-698)

Le pectoral matérialise cette union du 3 et du 4, par les quatre rangées de trois pierres qui le composent. Chacune des quatre rangées possède un sens : la prudence, la force, la justice et la tempérance sont les quatre vertus cardinales du chrétien. Une telle équivalence entre les vertus cardinales et les gemmes se retrouve à 
l'ouverture du deuxième livre du Livre du Trésor de Brunet Latin. Dans le texte encyclopédique, chaque vertu est représentée par une gemme, et non pas par un groupe de gemmes, sans aucune correspondance avec la classification du lapidaire mixte : la prudence est symbolisée par l'escarboucle ; la tempérance par le saphir ; la force par le diamant ; la justice par l'émeraude.

Cist ensegnemens sera sor les .iiii. principaus vertus. Dont la premiere est prudence, ki est segnefiee par le carboncle, ki alume la nuit et resplendist sour toutes pieres. La seconde est atemprance, ki est segnefiee par le saphir, ki porte celestial coulor, et est plus gracieuse que piere du monde. La tierce est force, ki est segnefiee par le diamant, ki est si fort k'il ront et perce toutes pieres et tous metaus, et por poi il n'est chose ki le puisse donter. La quarte vertu est justice, ki est segnefiee par l'esmeraude, ki est la plus vertuouse et la plus bele chose que oil d'ome puisse veoir ${ }^{19}$.

À cette lecture globale (les douze gemmes), puis par rangée (par groupe de trois gemmes) répond le niveau individuel de la gemme. L'article sur la sardoine superpose par exemple différentes gloses numériques :

La Bible et seint Jehans nos dit sarde grenaz ou parement

Aaron fu premierement ; ce fu la premiere nomée des .XII. et la meins renomée. /.../

Seint Jehans en Apocalipse nos dit que bien li fu avis que sardes fu en Paradis ou siste fondement veüz, por Adan qui fu deceüz et fu formez au siste di, (et) por le sanc du vendredi, que Dieus seigna por ses amis, fu li sardes grenat la miz. (v. 706-710 \& 728-736)

Première gemme du pectoral, elle est donnée comme la moins précieuse - ce qui laisse entendre que les pierres seraient rangées par ordre croissant de valeur. Sixième gemme de la Jérusalem céleste, elle rappelle le vendredi saint, sixième jour de la semaine. La rangée

19. Li Livres dou Tresor, édition critique par F. J. Carmody, Slatkine Reprints, Genève, 1975, Livre II, p. 175. 
et la place de la gemme sur cette rangée sont parfois aussi commentées, comme pour l'agate, deuxième pierre de la troisième rangée :

.II. choses ont senefiables :

por ce fu seconde monté(e)

en la .III. ce tire et contée.

Acate fu en tierce tire

por le tierz tens de baptetire

que Jhesu Crist et seint Jehans

preescherent /.../

(v. 1086-1092)

Le va-et-vient entre les textes de L'Exode et de L'Apocalypse de saint Jean est parfois entravé : en effet, les gemmes qui ornent le pectoral ne sont pas exactement les mêmes que celles de la Jérusalem céleste ${ }^{20}$ :

\begin{tabular}{|c|c|c|}
\hline Rang & $\begin{array}{c}\text { Pectoral d'Aaron } \\
\text { (Exode, XXVIII, 15-20 } \\
\text { \& XXXIX, 8-13) }\end{array}$ & $\begin{array}{c}\text { Jérusalem céleste } \\
\text { (Apocalypse, XXI, 19-20) }\end{array}$ \\
\hline 1 & sardoine & jaspe \\
\hline 2 & topaze & saphir \\
\hline 3 & émeraude & émeraude \\
\hline 4 & rubis & (sardonyx) \\
\hline 5 & saphir & sardoine \\
\hline 6 & jaspe & chrysolite \\
\hline 7 & ambre & béryl \\
\hline 8 & agate & topaze \\
\hline 9 & améthyste & (chrysoprase) \\
\hline 10 & chrysolite & (hyacinthe) \\
\hline 11 & onyx & améthyste \\
\hline 12 & béryl & \\
\hline
\end{tabular}

20. En gras figurent les gemmes qui apparaissent sur le pectoral d'Aaron et pas dans la Jérusalem céleste. Les gemmes de la Jérusalem céleste qui n'apparaissent pas sur le Pectoral d'Aaron sont mises entre parenthèses. 
Du point de vue numérique, la symétrie entre les textes bibliques comporte donc des failles, que le clerc parvient à résoudre très habilement, en remplissant les vides de sa démonstration. Le premier hiatus apparaît avec le rubis, absent de la Jérusalem céleste. Selon le clerc, l'absence du rubis métaphorise l'omniprésence de Dieu, Lui qui est partout sans s'arrêter nulle part :

Seint Jehans en sa glose dit que li biaus rubi pas ne vit ou haut fondement precieus car li verai Deus gracieus est entre ses amis tojors, nus ne set nus de ses sejorz en .I. eure en cor (autre) ou meleu ; einsi n'a point de propre lieu fors la ou li plest demorer, par tot doit l'en Dieu aorer. (v. 921-930)

Pour les autres pierres absentes de la Jérusalem céleste (l'ambre, l'agate, l'améthyste et l'onyx), le clerc n'apporte aucune justification $^{21}$. Pour l'améthyste, il va jusqu'à omettre toute notation numérique - et c'est là une exception notable - pour se concentrer sur la couleur pourpre de la gemme :

Amestice a porpre semblance ;

semblance a en seinte escripture

a la seintime vesteüre

dont Juïf Jhesu Crist vestoient

quant d'escorgies le batoient.

De porpre color le vestirent,

quant d'escorgies le batirent :

rois le fesoient par eschar,

de porpre covrirent sa char.

D'icel color vestir devroient

li roi quant haute cort tendroient ;

Salemon d'icele vestoit.

Amestice nos ramentoit

ce que je vos ai devant dit,

et si nos ramentoit l'abit

21. Notons que dans les autres manuscrits, ces quatre pierres sont étudiées à la fin du lapidaire : le clerc présente leurs vertus païennes, mais ne leur confère aucune vertu chrétienne. Pour le détail du texte, voir l'édition Pannier, v. $1305-1554$. 
de la gloriouse Marie,

et des anges la seignorie ;

la mort des martirs amentoivent

amestice, amentevoir doivent

ce que je vos ai ci conté.

(v. 1100-1119)

Pour chaque pierre, la glose s'appuie sur la symbolique des couleurs - prédilection pour le sens de la vue, qui fait écho au goût du Moyen Âge pour la lumière et les couleurs. Qu'elle soit monochrome ou polychrome, la gemme s'enrichit de multiples senefiances par le truchement des couleurs. Une seule couleur peut engendrer plusieurs gloses : ainsi la rougeur du sarde évoque à la fois la terre avec laquelle Dieu façonna Adam, nos péchés et le sang du vendredi saint (v. 711-736); les gemmes multicolores présentent le plus souvent une glose par couleur, à l'instar de l'agate noire et blanche :

Noire est, et i a une branches, que nature i mist, qui sunt blanches ; et cele petite noireur senefie la grant tristeur qu'avoir devons por noz pechiez, quar chacuns en est entechiez, por ce devons estre en tristece.

Li blans senefie hautece, es livres selonc le devis, es hauz arbres de Par(e)vis qui portent les fruiz pardurables. (v. 1075-1085)

La blancheur de l'onyx, qui évoque un ongle d'homme, est le point de départ d'une hiérarchie fondée sur un triple parallélisme 22 :

la glose Moysi por voir

dit de ceste oniche la some,

que il resemble ongle d'ome.

A celi senefie et monte

22. L'étymologie et la mythologie apparentent l'onyx à l'ongle, comme le rappellent P. Bariand et J.-P. Poirot : « À l'aide d'une pointe de flèche, Cupidon avait un jour coupé les ongles (onux) de Vénus endormie ; il laissa tomber sur le sable ces chutes d'ongles, que les Parques métamorphosèrent en pierre : car ce qui provient d'un corps céleste ne doit pas périr », Larousse des pierres précieuses, Paris, Larousse Bordas, 1998, article onyx, p. 192. 
qui molesce de char seurmonte : et ausi com ongle est plus dure de mole char et plus endure, mes n'ateint pas a la durece de la pierre ; aussi la molece de char sormonterent li seint, mes ne di pas qu'il venisseint a la grant durece des pierres au deable, c'est li pechierres qui endur(c)ist en son pechié par son faus cuor mal entechié.

(v. 1208-1222)

Plus dur que la chair, plus tendre que la pierre, l'ongle emblématise le saint, qui se situe entre la faiblesse de l'homme et la dureté du diable.

\section{LA GLOSE, DU LAPIDAIRE AU BESTIAIRE}

L'analogie se densifie également quand le lapidaire reprend les propriétés païennes décrites dans la première partie, notamment celles de l'émeraude et de l'ambre. Les textes patristiques et encyclopédiques ont répandu la légende de la récolte des émeraudes par le peuple des Arimaspes, qui doivent combattre les griffons, légende reprise dans la première partie du lapidaire mixte (v. 205-220) et relue selon l'exégèse spirituelle dans la seconde partie ${ }^{23}$ :

Seint Jehan nos dit en son livre,

en l'Apocalipse a delivre,

que cil qui a la foi entendent

qui ne puet faillir, et i tendent,

n'unt c'un ueil, c'est au roi d'amont

qui de bien fere nos semont ;

cil senefie a la gent

que je vos devisai devant,

qui se conbatent aus gripons,

Arimipiles est leur nons.

Chacuns gripons, n'est mie fable,

Si senefie le deable,

23. Voir l'article de Gérard Cames, "Or, émeraudes et griffons », dans Gazette des Beaux-Arts, 119e Année, Paris, octobre 1977, p. 105-108. 
qui est angoisseus par defors

de tolir nos la foi du cors,

(v. 851-864)

Les Arimaspes, dont l'œil unique représente Jésus Christ, sont les vrais croyants et vont chercher l'émeraude, symbole de la foi ; ils doivent lutter contre les griffons, représentations des diables. En glosant ces récits, le clerc les présente comme des symboles chrétiens, et non plus comme des réalités païennes; il en est de même pour l'ambre (v. 436-448). Considérée comme de l'urine de lynx, l'ambre est donc secrétée par les reins, siège de la luxure ; extériorisée et minéralisée, cette substance incarne désormais son contraire (selon le principe de l'analogie réversible), c'est-à-dire la chasteté :

\section{/.../ or si disons}

selonc la Bible et devisons, ou les estoires sunt segures, chastée porte le ligure, ce senefie par droiture. (v. 1033-1037)

Beste orientale, le lynx est un monstre, au sens étymologique du terme : il est donné à voir pour faire sens. Le lapidaire l'apparente au bœuf, symbole de l'évangéliste Luc, et aux prêcheurs :

Ces bestes, bien ce sachiez vos, dont chascune la terre esfondre por mestre ligure et repondre, senefient aus buoes qui errent la terre Jhesu Crist et perent par seinte predication, c'est leur signification. (v. 1052-1058)

Prenant la gemme comme point de départ, la glose s'étend à d'autres créatures : le lapidaire cède alors la place, au détour de quelques vers, au bestiaire, relayé par les décorations marginales, où apparaissent des créatures anthropomorphes et zoomorphes, le plus souvent hybrides. Dans la deuxième partie du lapidaire, un homme à l'épée décore la lettre ornée de l'émeraude, un combat opposant deux hommes à un dragon orne celle de la chrysolite (voir reproductions ci-après des folios $24 \mathrm{v}^{\circ}-25 \mathrm{r}^{\circ} \& 39 \mathrm{v}^{\circ}-40 \mathrm{r}^{\circ}$ ). Dans quelle mesure ces dessins sont-ils une glose du texte ? La thématique 
du combat y est récurrente : les créatures mi-humaines brandissent épées et massues, affrontent des monstres, etc. Peut-on y lire une figuration du bien en lutte contre le mal, en écho à la senefiance des pierres ? Cette lecture est d'autant plus tentante que les couleurs prédominantes sont le bleu, le rouge et l'or, couleurs mariales et royales. La distribution des motifs reste néanmoins aléatoire dans le manuscrit, sans changements notables entre les deux parties du traité. Par ces décorations qui s'étirent dans les marges, le décorateur fait écho aux bouts de ligne qui terminent le vers. Soucieux de créer une unité picturale et chromatique, le dessinateur semble certes avoir voulu évoquer le sens du texte, mais tout autant en égayer la lecture par des drôleries ${ }^{24}$.

La binarité du lapidaire chrétien opère, grâce à l'omniprésence de Dieu dans le traité, un syncrétisme entre les vertus païennes et chrétiennes. Un jeu de miroir s'établit entre les deux parties, à l'image de l'opposition entre l'Ancien Testament et le Nouveau Testament. La seconde partie se présente en effet comme une relecture de la première, une glose qui dépasse et transcende les croyances anciennes, tout en s'appuyant sur les auctoritates bibliques et patristiques. Il ne faut cependant pas se méprendre sur la vocation didactique du lapidaire : seuls les clercs pouvaient véritablement comprendre cette glose, fondée sur des connaissances théologiques précises. Léopold Pannier précise que « la signification mystique, les légendes symboliques que les Pères avaient attachées aux pierres du Rational restèrent presque toujours lettres closes pour quiconque ne faisait pas partie de l'Église. Toutes ces moralisations n'étaient en quelque sorte qu'un langage réservé aux seuls initiés et compris par eux seuls $»^{25}$.

Au fil des vers se dessinent les contours du poète anonyme : ce clerc érudit connaissait aussi bien les traités de mystique que les

24. Définition de Denis Muzerelle, «scène de fantaisie plus ou moins comique, sans rapport avec le texte, incluse dans la décoration », Vocabulaire codicologique. Répertoire méthodique des termes français relatifs aux manuscrits, Paris, CEMI, 1985 (Rubricae I), p. 512.

25. Les lapidaires français du Moyen Age des XII ${ }^{e} \mathrm{XIII}^{e}$ et XIV siècles, op. cit., p. 216. 
lapidaires anciens et contemporains. Cet homme fait de nombreuses références au prêche et aux prêcheurs et témoigne d'une communauté de pensée avec Albert le Grand : il s'agissait peut-être d'un Dominicain. Par le truchement de ce traité aux accents mystiques, le poète-théologien a traduit son élan vers Dieu. Considérant la nature et ses créatures comme une théophanie permanente, il a tenté d'accéder, selon la célèbre formule de saint Paul, per visibilia ad invisibilia.

Université de Provence

29 Avenue Robert Schuman

13621 Aix-en-Provence Cedex 01 
